\title{
Automated heartsound analysis/Computer-aided auscultation: A cardiologist's perspective and suggestions for future development
}

\author{
C. Becket Mahnke
}

\begin{abstract}
Heart disease is a major cause of worldwide morbidity and mortality. Properly performed, the cardiac auscultatory examination (listening to the heart with a stethoscope) is an inexpensive, widely available tool in the detection and management of heart disease. Unfortunately, accurate interpretation of heartsounds by primary care providers is fraught with error, leading to missed diagnosis of disease and/or excessive costs associated with evaluation of normal variants. Therefore, automated heartsound analysis, also known as computer aided auscultation (CAA), has the potential to become a cost-effective screening and diagnostic tool in the primary care setting. A cardiologist's suggestions for CAA system design and algorithmic development are provided.
\end{abstract}

\section{BACKGROUND AND SIGNIFICANCE}

$\mathrm{H}$ EART disease is a major cause of morbidity and mortality across all ages throughout the world. In adults, heart disease remains the most common cause of death in developed countries [1]. In children, heart malformations represent the most frequently encountered birth defect [2]. Finally, rheumatic heart disease remains a major public health issue for developing nations [3]. In most cases, auscultation of the heart with a stethoscope is often the first screening tool utilized by primary care providers.

In addition to the stethoscope, physicians have a variety of other methods available to evaluate cardiac pathology to include cardiac ultrasound (echocardiography), cardiac catheterization, computed tomography (CT) and magnetic resonance imaging (MRI). Although each is a powerful tool in high-quality cardiac care, all are limited to some degree by patient safety, limited availability, and cost. Even with the availability of new low cost, portable ultrasound devices, echocardiography still requires highly trained personnel for data acquisition and interpretation. Therefore, an accurately performed auscultatory examination remains the most costefficient screening tool for many forms of cardiac disease.

However, multiple researchers have demonstrated poor auscultatory skills among primary care providers, exactly those tasked with detection of cardiac disease in an early state [4-6]. This is not entirely surprising, as just a fraction of the acoustic energy generated by a beating heart is

Manuscript received April 7, 2009. This work was supported in part by the Telemedicine and Advanced Technology Research Center's Army Advanced Medical Technology Initiative (AAMTI) program. The views expressed in this manuscript are those of the author and do not reflect the official policy or position of the Department of the Army, Department of Defense, or the U.S. Government.

C. Becket Mahnke is with Tripler Army Medical Center, Pediatric Department (Cardiology), 1 Jarrett White Rd, Honolulu, HI 96859 USA (phone: 808-433-6225;e-mail: Christopher.Mahnke@us.army.mil). detectable by the human auditory system [7]. Misinterpretation of the auscultatory examination results in missed opportunities to fully investigate cardiac pathology, with potentially poor patient outcomes. Alternatively, referral of normal or "innocent" findings for further evaluation results in excessive healthcare expenditures and unnecessary patient stress [8-11].

In contrast, cardiologists can often accurately determine normal from disease states based on auscultation alone [1214]. Prior to the invention of advanced imaging, the auscultatory examination was the cardiologist's primary tool in the diagnosis of heart disease. Skilled cardiologists had to accurately determine which sounds were truly abnormal to ensure that patients with normal variants did not undergo risky invasive procedures. In addition, heartsound recordings, called phonocardiograms (PCGs), were made in order to improve diagnostic accuracy through visual inspection and analysis. A typical PCG recording included 4-6 channels, consisting of 1-2 audio channels and a various combination of electrocardiogram, carotid/jugular pulse waveforms, and an apexcardiogram. Recorded simultaneously onto paper, these were manually examined for the determination of disease presence and severity [15]. However, cardiologists of that era did not have the powerful computational tools available for complex signal analysis available today, limiting phonocardiography's widespread clinical use. Combined with the introduction of advanced, non-invasive imaging techniques, phonocardiography fell out of clinical practice.

Given 1) the difficulties in acquiring and maintaining auscultatory competence, 2) the presently available computational tools for complex signal analysis, and 3) the need for an inexpensive screening tool for cardiac disease, there is tremendous potential for a system of automated heartsound analysis, also known as computer aided auscultation (CAA). In the United States, $>5$ million people have diagnosed heart failure, and 550,000 incident cases occur annually, accounting for $>1$ million hospitalizations and 285,000 deaths each year. The annual direct and indirect costs of heart failure are estimated to exceed \$33 billion annually in the United States [1]. S3 and S4 heartsounds are an early abnormality in heart failure patients, yet difficult to hear and frequently missed by healthcare providers due to their frequency characteristics [4]. However, S3 and S4 sounds may be detectable by CAA, leading to potentially better outcomes via earlier medical intervention [16]. Furthermore, serial evaluation via CAA, even at home, could guide individualized, real-time therapy decisions. Also, 
CAA could be used as an initial screening tool in heart murmur evaluation, a common reason for cardiology referral. Pre-participation screening sports physicals examinations could be augmented with CAA to improve identification of those at risk for exercise related sudden death [17]. Rheumatic heart disease, the most common form of cardiac disease in most of the world, has recently been show to be underappreciated by an order of magnitude by auscultatory exam alone [18]. However, such patients may indeed have acoustic findings, suggesting that a properly designed and sufficiently sensitive CAA system may be able to detect those with "silent" rheumatic heart disease who would benefit from early therapy [19]. Other imaging modalities (echocardiography, cardiac catheterization, CT/MRI) could no doubt address each of the above situations, but limited availability and high cost make this approach untenable from an economic perspective.

\section{Computer Aided Auscultation (CAA)}

While others have demonstrated the potential utility of automated heartsound analysis [20-23], CAA has yet to make an impact on routine cardiac care. The reasons for this are multi-factorial and each must be addressed if CAA is to become routinely endorsed by healthcare providers and patients. A successful CAA system will require both a heartsound recording device capable of acquiring highquality datasets and PCG interpretation algorithms that are highly sensitive and specific. Given the many challenges, CAA system development is likely to require collaboration between engineers, clinicians, and industry partners.

Successful CAA system development first requires a clear definition of its ultimate intended use, be it either as a screening device (disease present/absent) or a complete cardiac diagnostic tool (disease type and severity). If intended as a screening device for detection of cardiac abnormalities requiring referral for further cardiac evaluation, algorithms are likely to be significantly simpler to design and implement. In this logical first step, CAA system development should focus on highly accurate identification of normal auscultatory findings, with cardiology referral for anything falling outside a predetermined range. Once accomplished, further CAA refinements can focus on disease specific diagnosis followed by severity determination algorithms.

From a clinical standpoint CAA must overcome a variety of hurdles. First, the PCG recording system must be easy to use by minimally trained personnel in a primary healthcare setting. Sound sensors should be adhesive to allow for precise positioning, low cost or reusable, and provide a relatively flat response curve across physiological frequencies. The waveforms for each data channel should be displayed in real time during acquisition, providing immediate feedback to the recording technician thus ensuring high quality data for subsequent analysis. PCG datasets should be stored for local and/or remote physician review in both bell and diaphragm modes with a clinically intuitive interface (Fig 1). In addition, this PCG data must be in a format that can integrate into the electronic medical record for documentation purposes and serial evaluation over time. The CAA analysis must be performed real-time to allow for immediate patient disposition (normal vs. refer for additional testing). Finally, PCG analysis must once again become a reimbursed medical procedure in order to fund system procurement, use, and maintenance.

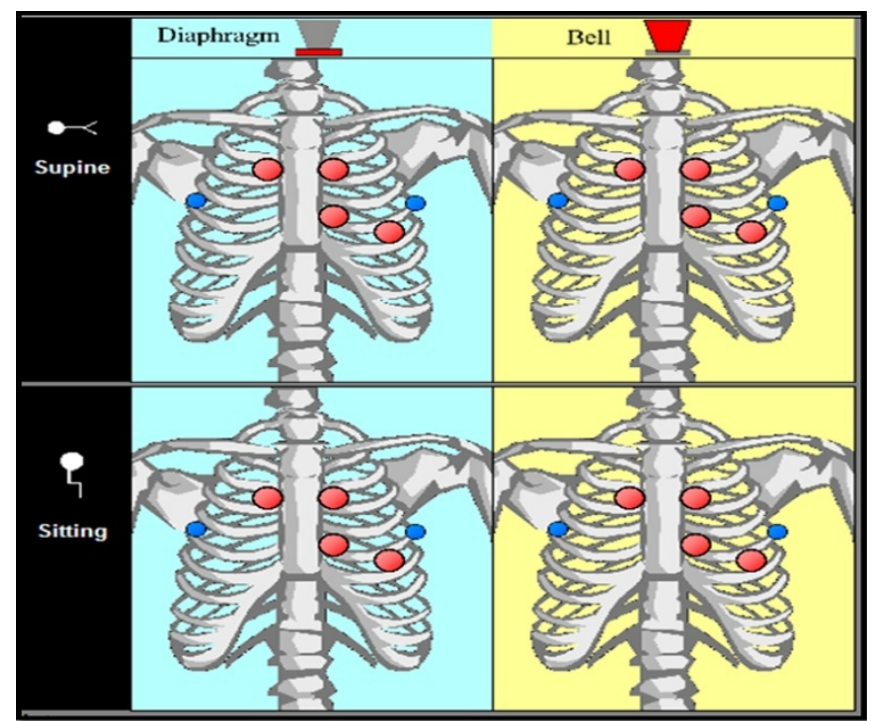

Fig. 1. Graphical user interface allowing for clinically intuitive playback and review of recorded heartsounds. When a colored circle is selected, the corresponding recorded heartsound from that chest location is played. The reviewer can listen with either the bell or diaphragm in both supine and sitting position, as is commonly practiced in a complete auscultatory examination.

From a technical standpoint, an open-access audio standard for medical data should first be established. Currently there is no uniformity or standardization in today's electronic stethoscope market, resulting in PCG datasets with varying recording characteristics (sample rate, frequency response), file formats, and filtration methods. Such variability limits research collaboration and inhibits integration into the electronic medical record. Medical imaging technology faced similar challenges, but these were largely eliminated with the Digital Imaging and Communications in Medicine (DICOM) standard, which allows for easy sharing across both clinical and research platforms.

To be clinically acceptable, CAA algorithms must have sufficiently high sensitivity and specificity so as to minimize both false positive and negative interpretations. Such endeavors face significant challenges based on both the complexity of the physiologic signals as well as the inherent variation of normality and disease across the population. Nevertheless, trained cardiologists routinely determine cardiac disease presence, type, and severity with the auscultatory examination, demonstrating that such accuracy is attainable [12-14]. One key element is that the cardiologist performs a complete auscultatory examination, listening 
with both bell and diaphragm at multiple chest locations throughout the cardiac and respiratory cycle. In contrast, nearly all CAA research has focused on analysis of a short duration single site PCG recording that does not span the entire respiratory cycle. PCG recording site and patient position, critical elements in a cardiologist's evaluation, are often ignored as well. The single site CAA approach is akin to asking the cardiologist to limit his auscultatory examination to only one site on the chest, in an unknown patient position, and for a very limited time period. Finally, the single site CAA approach requires the end-user to determine the most important site for PCG acquisition. Since the intended end-user of a CAA system is not a cardiac expert, the single site approach will likely result in suboptimal data for analysis. Given all the reasons above, successful CAA will likely require a multi-site approach which also incorporates patient position and sufficiently long recordings (Fig. 2).

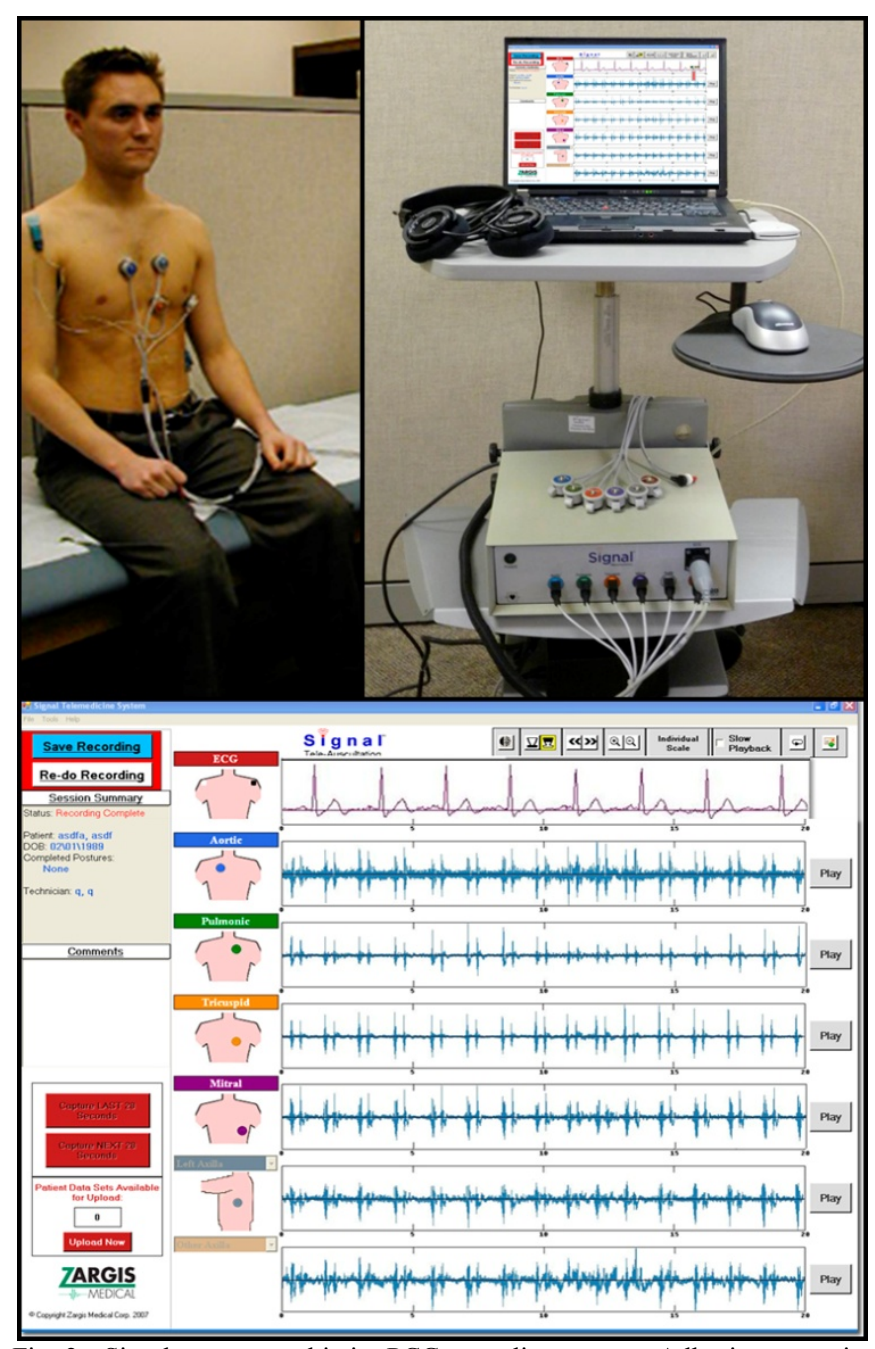

Fig. 2. Simultaneous, multi-site PCG recording system. Adhesive acoustic sensors allow for precise positioning and various patient postures (top left). Portable cart-based system with voice-guided prompts for easy clinical use (top right). Graphical user interface with real-time signal display of electrocardiogram, plethysmography, and six acoustic sites to ensure highquality data acquisition (bottom).
While the cardiologist gathers heartsound data sequentially from each site, PCG recording systems can acquire multi-site data either sequentially (single sensor) or simultaneously (multiple sensors). However, simultaneously acquired PCG recordings have the benefit of intrinsic time alignment of all recorded channels and significantly reduced data acquisition time. Since all PCG signals are timealigned, key timing and intensity variations of various cardiac events can be compared from the multiple recording sites. It is anticipated that the use of time-aligned datasets will improve the CAA accuracy because the results from a single PCG signal can be cross-referenced with those obtained from other sites, allowing for corroboration and/or arbitration of confusing and/or conflicting results. The use of simultaneously acquired PCG analysis also promises opportunities for new forms of CAA analysis, beyond emulating current diagnostic procedures. By examining the characteristics of PCGs at several auscultation sites simultaneously, new diagnostically relevant patterns may emerge. In addition to improving the performance of automated systems, the insights these patterns provide may lead to new clinical techniques.

In addition to acoustic recordings, a CAA system can benefit from the incorporation of other sensor modalities which can provide additional data for analysis. The electrocardiogram, arterial and venous pulsations, and apexcardiogram can be acquired simultaneously with the addition of appropriate sensors. Such data will provide additional channels for further corroboration of conflicting results, and were originally utilized in manual PCG analysis for disease specific identification and quantification [24].

Furthermore, the current CAA approach often fails to leverage the physiologic principles that guide cardiologists during their clinical evaluation. The cardiac system has physiologic limits with regard to timing of events such as systole and diastole, and knowledge of these events can be exploited to improve accuracy. For instance, labeling of an event known to have a subsequent event (i.e., S1 followed by S2) could be optimized by knowing when to "look" for the subsequent event within the physiologic possibilities. This has the potential to speed the automated process by provide a blanking or skip area that may decrease computational requirements. Events occurring outside of physiologically possible time intervals can be ignored or at least not falsely identified. In addition, clinical characteristics of various auscultatory events can be optimized to further limit computational requirements. For example, the presence of an ejection click of bicuspid aortic valve can be analyzed first at the typical auscultatory location. If not present, analysis for this finding can be skipped from the analysis of other channels. Finally, disease specific diagnostic algorithms should be modeled on clinical decision algorithms, incorporating a combination of auscultatory parameters, such as characteristics of each heart sound, murmur qualities, and presence/absence of additional sounds (clicks, snaps, etc). 
With the preceding factors in mind, we have designed a PCG recording device that allows for simultaneous acquisition of 6 auscultatory sites along with a single channel electrocardiogram and a plethysmography waveform (Fig. 2; Signal, Zargis Medical, New Jersey). The plethysmography waveform provides a more patient-friendly surrogate for the carotid pulse waveform utilizing a standard, non-invasive pulse oximetry probe. The patient's position is captured and twenty second recordings are acquired to ensure capture of multiple cardiac and respiratory cycles. Datasets are uploaded to our telemedicine platform for remote physician evaluation utilizing a clinically intuitive graphical user interface and reporting system (Fig. 1). Signal analysis is not currently performed; however, the PCG datasets are being utilized for ongoing CAA algorithm development.

\section{CONCLUSION}

Computer aided auscultation (CAA), based on multi-site, simultaneously acquired heartsound recordings, has the potential to become an important cardiac screening and diagnostic tool if properly designed and implemented. Given the many challenges, CAA system development is likely to require collaboration between engineers, clinicians, and industry partners

\section{REFERENCES}

[1] W. Rosamond et. al. "Heart disease and stroke statistics-2007 update: a report from the American Heart Association Statistics Committee and Stroke Statistics Subcommittee," Circulation, 115:e69-e171, 2007.

[2] J.I. Hoffman, S. Kaplan, "The incidence of congenital heart disease," J Am Coll Cardiol, Vol 39, pp.1890-1900, 2002.

[3] J.R. Carapetis, M. McDonald, N.J. Wilson, "Acute rheumatic fever," Lancet, 366:155-168, 2005.

[4] S. Mangione, L. Nieman, "Cardiac auscultatory skills of internal medicine and family practice trainees: a comparison of diagnostic proficiency," JAMA, $278: 717-722,1997$.

[5] P. Gaskin, S. Owens, N. Talner, S. Sanders, J. Li, "Clinical auscultation skills in pediatric residents," Pediatrics, 105 :1184$1187,2000$.

[6] C.B. Mahnke, A. Nowalk, D. Hofkosh, J. Zuberbuhler, Y. Law, "Comparison of two educational interventions on pediatric resident auscultation skills," Pediatrics, 113(5):1331-5, 2004.

[7] J. Butterworth, M Chassin, R McGrath. Cardiac Auscultation. Grune \& Stratton: 1955.

[8] D. Danford, A. Nasir, C. Gumbiner, "Cost assessment of the evaluation of heart murmurs in children," Pediatrics, 91:365-8, 1993.

[9] R. Geggel, L. Horowitz, E. Brown, M. Parsons, P. Wang, D. Fulton, "Parental anxiety associated with referral of a child to a pediatric cardiologist for evaluation of a Still's murmur," J Pediatrics, 140(6):747-52, 2002.

[10] M. Xu, D. McHaffie, "Nonspecific systolic murmurs: an audit of the clinical value of echocardiography," $N Z$ Med J, 106(950), 54-56, 1993.

[11] A. Bloch, J Crittin, A. Jaussi, "Should functional cardiac murmurs be diagnosed by auscultation or by Doppler echocardiography?" Clin Cardiol, 24(12), 767-9, 2001.

[12] C. Roldan, B Shively, M. Crawford, "Value of the cardiovascular physical examination for detecting valvular heart disease in asymptomatic subjects," Am J Cardiol, 77(15), 1327-31, 1996.

[13] J. Smythe, O. Teixeira, P. Vlad, P. Demers, W. Feldman, "Initial evaluation of heart murmurs: are laboratory tests necessary?," Pediatrics, 86:497-500, 1990.
[14] B. McCrindle, K. Shaffer, J. Kan, K. Zahka, S. Rowe, L. Kidd, "Cardinal clinical signs in the differentiation of heart murmurs in children," Arch Pediatr Adolesc Med, 150:169-74, 1996.

[15] M. Tavel, Clinical phonocardiography and external pulse recording $\left(2^{\text {nd }} E d\right)$, Year Book Medical Publishers: 1972.

[16] P. Hult, T. Fjallbrant, B. Wranne, P. Ask, "Detection of the third heart sound using a tailored wavelet approach," Med Biol Eng Comput, 42(2), 253-8, 2004.

[17] M. Papadakis, G. Whyte, S. Sharma, "Preparticipation screening for cardiovascular abnormalities in young competitive athletes," $B M J$, 337:a1596, 2008.

[18] E. Marijon, D.S. et al. "Prevalence of rheumatic heart disease detected by echocardiographic screening," New England Journal of Medicine, 357(5):470-6, Aug 2007.

[19] C. Vongprateep, R. Lauer, A. Diehl, "Intraesophageal phonocardiography in the diagnosis of occult rheumatic mitral valve disease," Pediatrics, 38, 892-6, 1966.

[20] W.Thompson, C. Hayek, C. Tuchinda, J. Telford, J. Lombardo, "Automated cardiac auscultation for detection of pathologic heart murmurs," Pediatr Cardiol, $22: 373$-379, 2001.

[21] C. DeGroff et al, "Artificial neural network-based method of screening heart murmurs in children," Circulation,103:2711 -2716, 2001.

[22] R. Watrous, J. Bedynek, T. Oskiper, D. Grove, "Computer-assisted detection of systolic murmurs associated with hypertrophic cardiomyopathy: a pilot study," Texas Heart Institute Journal, 31(4):368-75, 2004.

[23] R. Watrous, W. Thompson, S. Ackerman, "The impact of computerassisted auscultation on physician referrals of asymptomatic patients with heart murmurs," Clinical Cardiology, 31(2), 79-83, 2008.

[24] J. Bonner Jr., H. Sacks, M. Tavel, "Assessing the severity of aortic stenosis by phonocardiography and external carotid pulse recordings," Circulation, 48(2), 247-52, 1973. 